Portland State University

PDXScholar

\title{
Meeting the Transition Needs of Urban American Indian/Alaska Native Youth through Culturally Based Services
}

\author{
Barbara J. Friesen \\ Portland State University \\ Terry L. Cross \\ National Indian Child Welfare Association \\ Pauline Jivanjee \\ Portland State University, jivanjee@pdx.edu \\ Ashley Thirstrup \\ Native American Youth and Family Center \\ Abby Bandurraga \\ Portland State University
}

See next page for additional authors

Follow this and additional works at: https://pdxscholar.library.pdx.edu/rri_facpubs

Part of the Social Work Commons

Let us know how access to this document benefits you.

\section{Citation Details}

Published as: Friesen, B.J., Cross, T.L., Jivanjee, P. et al. J Behav Health Serv Res (2015) 42: 191.

This Post-Print is brought to you for free and open access. It has been accepted for inclusion in Regional Research Institute by an authorized administrator of PDXScholar. Please contact us if we can make this document more accessible: pdxscholar@pdx.edu. 


\section{Authors}

Barbara J. Friesen, Terry L. Cross, Pauline Jivanjee, Ashley Thirstrup, Abby Bandurraga, L. Kris Gowen, and Jen Rountree 
[This manuscript was published online November 12, 2014 in the

Journal of Behavioral Health Services \& Research. The final publication is available at

Springer via http://link.springer.com/article/10.1007/s11414-014-9447-2]

\title{
Meeting the Transition Needs of Urban American Indian/Alaska Native Youth through Culturally Based Services
}

\author{
Barbara J. Friesen, Terry L. Cross, Pauline Jivanjee, Ashley Thirstrup, \\ Abby Bandurraga, L.K. Gowen, \& Jen Rountree.
}

\begin{abstract}
This article reports findings from three qualitative studies exploring supports for positive transitions of American Indian/Alaska Native (AI/AN) youth to adulthood. Community-based participatory methods were employed through a research partnership involving a culturally based community agency, the Native American Youth and Family Center (NAYA), the National Indian Child Welfare Association, and Portland State University. Studies utilized a Relational Worldview (RWV) framework, where well-being is understood as a balance among the domains of mind, body, spirit, and context. Collectively, findings demonstrate that NAYA employs culturally grounded interventions to overcome the traumatic histories and current oppressive conditions affecting lowincome urban AI/AN youth with mental health challenges and to support their well-being and transition to adulthood. In addition, addressing the mental health and well-being of AI/AN youth in culturally appropriate ways involves consideration of all RWV domains. Recommendations for behavioral health practice are to connect AI/AN youth to culturally specific services whenever possible, utilize cultural consultants, and implement holistic and positive approaches to mental health.
\end{abstract}

\section{Introduction}

...we will come to realize that "culture" is much more than participation in ceremony or fluency in traditional language - in fact, cultural practices comprise the almost invisible participation in shared thought and activity that need never be conscious since most people in the community are socialized into such routines. ${ }^{1}$

This article reports on findings from three studies that explore the transition needs of American Indian/Alaska Native (AI/AN) youth and examine the culturally based approaches and resources that address those needs. These investigations were conducted by a research partnership involving a culturally based community agency, Native American
Youth and Family Center (NAYA); the National Indian Child Welfare Association; and research staff from Portland State University. Within a community-based participatory research framework, the research team, consisting of representatives from each of the three partner organizations, undertook a series of steps to better understand the transition needs of AI/AN youth, and to expand the knowledge base regarding culturally based resources and services that can be used to address these needs.

A focus on AI/AN transition-aged youth is important for several reasons. Although AI/AN young people demonstrate many strengths and remarkable resilience, ${ }^{2,3}$ they also have poor mental health, substance use, and suicide outcomes relative 
to their non-Native peers. National data compiled by the Urban Indian Health Institute ${ }^{4}$ shows that urban $\mathrm{AI} / \mathrm{AN}$ youth experience risk factors relating to substance abuse and violence at rates double those of their white counterparts, and attempt suicide nearly three times more often than Caucasian youth. In the years 2004-2008, AI/AN students, along with African American students, were the top two most represented groups in special education, and AI/AN youth representation increased the most during this period. ${ }^{5}$ In addition, urban AI/ AN youth experience poverty and drop out of high school at significantly higher rates than their urban, white peers, and are at greater risk for serious mental health problems. ${ }^{4}$

The impact of historical trauma related to colonization, genocide, dislocation, and forced assimilation through placement of Indian children in foster care and/or boarding schools and other strategies has been described by many Native American authors. ${ }^{6,7}$ Recent research addresses links between historical trauma and current problems, ${ }^{8}$ and also provides evidence for the intergener- ational transmission of historical trauma. ${ }^{9}$ Many AI/AN youth also suffer from trauma related to witnessing violence, and experiencing physical or sexual abuse, discrimination, and poverty. ${ }^{9}$ Thus, for $\mathrm{AI} / \mathrm{AN}$ youth, preparing for the future also involves addressing cumulative, collective historical trauma as well as dealing with trauma related to the negative and hurtful events they have experienced.

$\mathrm{AI} / \mathrm{AN}$ young people have poor transition outcomes relating to education, employment, and poverty, ${ }^{10}$ and AI/AN young people transitioning from foster care into adulthood struggle with transition outcomes disproportionately compared to non-Native peers. ${ }^{11}$ Although a few articles address issues faced by AI/AN young people on reservations when they leave high school, ${ }^{12-14}$ little is known about transition for urban AI/AN youth, especially those with mental health concerns.

Given the documented issues affecting AI/ AN emerging adults, it is important to examine their transition needs within the context of their culture(s) so that culturally appropriate approaches can be implemented and/or developed to minimize risk and increase protective factors among this population. Shafer and Ramasamy ${ }^{14}$ suggest that the concept of transition itself may be based on the values of an urban, Anglo culture, especially with regard to gainful, competitive employment and emancipation from the family home: "...the values of cooperation, interdependence and communal responsibility and action often conflict with the values of independence and competition that are often implied by transition services" (p. 61)

Leake and colleagues ${ }^{15}$ address possible implications for transition planning related to cultural differences. They contrast mainstream values, which are largely individualistic, with the collectivistic orientation of many other groups including AI/ AN youth, and describe the different skills sets that go with each orientation. Long, et al. ${ }^{11}$ also compare the mainstream transition emphasis on independence and emancipation with the AI/AN values of interdependence and note the protective factors of extended family, community, and tribal relationships. Cultural differences that may affect transition planning are summarized in Table 1.

These contrasting positions not only reflect differences in values, but are also built into laws and regulations that reflect the values of the larger society. For example, young adults who receive housing assistance cannot have relatives living with them. However, Native youth stand to lose family support and critical cultural and social capital if they follow this rule and do not allow homeless family members to move in with them. The Native youth that gains status in his family for assuming cultural roles in subsistence activities or tribal ceremonies may miss school events, thus delaying graduation and/ or sacrificing grades. For AI/AN youth, making a transition from dependency to providership is essential for the health of the collectivist culture, making interdependence more important than independence. ${ }^{16}$ Another example of this tension can be seen in graduation ceremonies. In independenceoriented cultures, a graduation recognizes individual accomplishment. In interdependence-oriented cultures, graduating honors the group. Some Native youth and families resolve this tension by attaching an eagle feather to a mortar board, grounding the 
Table 1. Cultural differences that may affect transition planning for AI/AN youth

\begin{tabular}{|c|c|c|}
\hline Dominant Culture & Al/AN Culture & \\
\hline Individualistic orientation & Collectivistic orientation & Sources \\
\hline Transition emphasis/goals & Transition emphasis/goals & Leake, et al. ${ }^{10}$ \\
\hline Self-determination, individual choice & Group or hierarchical decision-making & $\begin{array}{l}\text { Long, et. al }{ }^{6} \\
\text { Shafer \& Ramasamy }\end{array}$ \\
\hline Competitiveness, personal achievement & $\begin{array}{l}\text { Cooperation, group competitiveness, and } \\
\text { achievement }\end{array}$ & Leake, et al. ${ }^{10}$ \\
\hline Post-secondary education & $\begin{array}{l}\text { Contributing to family through wages, } \\
\text { housework }\end{array}$ & Leake, et al. ${ }^{10}$ \\
\hline $\begin{array}{l}\text { Independent living and self-reliance, } \\
\text { emancipation }\end{array}$ & $\begin{array}{l}\text { Residing with kin, interdependence, and } \\
\text { (for students with disabilities) possibly being } \\
\text { cared for }\end{array}$ & Leake, et al. ${ }^{10}$ \\
\hline Creating a transition plan on paper & $\begin{array}{l}\text { Establishing personal relationships between } \\
\text { professionals, youth, and families }\end{array}$ & Leake, et al. ${ }^{10}$ \\
\hline
\end{tabular}

celebration in a collective accomplishment rather than that of an individual. Many youth experience an almost irreconcilable tension trying to have a foot in each world. The demands of transition in the mainstream world, by necessity, separate them from the expected transitional tasks of their own culture.

\section{Relational Worldview as a Framework for Research}

The three studies of transition needs and culturally based practices were conducted using a Relational Worldview (RWV) framework. ${ }^{16-18}$ The RWV perspective can be used by Indians and non-Indians to understand culture as a resource for working with $\mathrm{AI} / \mathrm{AN}$ youth ${ }^{16}$ and provide a holistic and culturally grounded framework for research and practice with AI/AN children and families. In the RWV, well-being is understood as a balance among four elements of life often depicted as quadrants of a circle including mind, body, spirit, and context. ${ }^{18}$ "Context" includes relationships with adults, elders, family, peers, and school, and many other external factors. It is the arena in which social functioning plays out. "Spirit" refers to the spiritual nature of the human experience and is closely associated with a sense of meaning, hope, faith, moral values, and one's relationship with a higher power. "Mind" refers to all conscious intellectual functioning as well as emotional functioning. "Body" refers to all physiological functioning ranging from genetics and brain chemistry to nutrition and sleep. The four quadrants are seen as all inclusive and interdependent, and conditions in any one of the four areas are assumed to influence all of the other quadrants. Within this framework, well-being is seen as the "sum total, at a moment in time, of these complex interactions." 16 Living by culturally based values such as reverence for elders, self-respect, gratitude, and avoidance of negativity is thought to balance the quadrants thus sustaining a healthy life even in the face of adversity. By measuring community-defined outcomes in each quadrant, and examining changes over time, project researchers are working to identify culturally grounded evidence of effectiveness. The research team is also examining overall balance at the program level by examining youth outcomes across the quadrants.

In a previous study involving elders, young people, families, and community representatives, ${ }^{19}$ members of NAYA's community identified a set of outcomes associated with "successful Native 
American youth." Organized within the RWV, these outcomes include healthy relationships, safety, positive community relationships and contributions, and connecting with resources (Context); coping, personal qualities and capacities, education, employment, focus and determination, cultural knowledge, and identity (Mind); healthy lifestyle, fitness, health care, housing, and finances (Body); and spiritual understanding and practices, connections to Native ancestry, knowledge and skills in traditional cultural practices, balance, and expressing Native identity (Spirit). These outcomes were then incorporated into an online youth self-assessment tool ${ }^{20}$ that serves as the basis for service and program planning and evaluation.

Across the focus groups, there was a strong emphasis on the importance of youth achieving a positive cultural identity; this goal is reflected in NAYA's mission, "to enhance the diverse strengths of our youth and families in partnership with the community through cultural identity and education." ${ }^{21}$ Research evidence supporting the relationships between positive cultural identity and a number of desirable youth outcomes that include reduced prevalence of suicide at the community level, ${ }^{22}$ school success, ${ }^{23-25}$ increased school belongingness, ${ }^{25}$ stronger adherence to anti-drug norms, ${ }^{26-28}$ and higher self-esteem. ${ }^{26}$ Additional outcomes that are positively associated with cultural identity are higher social functioning, ${ }^{25}$ increased resilience and better psychological health, ${ }^{28}$ and positive physical health. ${ }^{28-30}$

Efforts to explain the theoretical basis for relationships between positive cultural identity and desirable outcomes vary; however, several authors address the importance of youth's placing themselves within a historical perspective and understanding how the past, present, and future are tied together. In her essay addressing the "importance of identity, history, and culture in the well- being of indigenous youth," ${ }^{11}$ Wexler proposes that "if young people can make sense of their experiences by locating themselves and their situation within historical understanding and community meaning, they are better able to overcome hardship and sustain psychological health". (p. 271) Gitxsan First Nations scholar
Blackstock's breath of life theory ${ }^{32}$ builds on the RWV with expansive concepts of time and reality. Blackstock points out that one of the most fundamental differences between First Nations and nonAboriginal ontology relates to concepts of time. The seven generations concept of time, where the past, present, and future are mutually reinforcing, means that one's actions are informed by the experience of the past seven generations and by considering the consequences for the next seven generations. Chandler and Lalonde's ${ }^{22}$ continuity theory suggests that identity development involves having a sense of self as continuous through the past, present, and future, and that this continuity is especially important in reduction of suicide risk.

\section{Description of the Study Setting}

The study site is a culturally and community-based agency that provides services to selfidentified AI/AN youth and young adults and their families in a three-county area. An estimated 40,783 $\mathrm{AI} / \mathrm{AN}$ people live in the three-county area; more than 13,000 are under 18 years of age. ${ }^{33}$

NAYA provides a broad array of services to AI/ AN young people and families to address the needs of the urban AI/AN community. These include educational, cultural arts, recreational, domestic violence, foster care, and independent living programs; as well as housing and home ownership assistance, and mental health and case management services. NAYA's approach is holistic and integrative, consistent with the RWV. ${ }^{16}$ It also reflects principles of Positive Youth Development (PYD) $)^{34,35}$ such as developing a positive identity, setting goals, making decisions, acquiring a sense of mastery, and developing prosocial connectedness, i.e., positive relationships and community-mindedness.

NAYA's preparation of young people for successful transitions includes some specific programming, such as a child welfare-funded independent living program for AI/AN foster care youth, and a number of education- and work-related opportunities and supports. In many ways, however, all of NAYA's many programs and activities are focused on helping youth develop a positive cultural identity, and more broadly on their healthy development 
so that youth can transition into a healthy adulthood. For example, youth may participate in the Youth and Elders Council that focuses on community advocacy. Youth, under the close mentorship of the elders, engage in community service and in so doing, learn skills, get approval (or correction) from respected individuals, build self-esteem, and gain a positive cultural identity. While this group is not labeled as a transition program, imbedded in it are essential elements of Native transition. In this council, youth are given responsibility both by and for the elders. Youth by their advocacy contribute to the well-being of the elders while benefiting from the guidance of an earlier generation. These real life lessons develop youth self-efficacy through their making a contribution to the well-being of the whole. The transition out of dependency toward interdependence facilitates youth achievement of culturally appropriate independence.

NAYA's emphasis on helping youth develop a positive cultural identity was developed by the parents, elders, and other community members who founded NAYA as a place for Native youth to have a safe and positive experience. The NAYA community's investment in the importance of a positive cultural identity is rooted not in evidence based on formal theory or research, but on wisdom generated by experience, observations, and a shared understanding of the history that forms the context for the development of AI/AN youth. The historical context influences the challenges faced by AI/AN youth before, during, and after their transition(s) from childhood to adulthood, from school to work, and to services and programs designed for adults. It also has shaped the interventions and support strategies used by NAYA staff and other members of the NAYA community.

The three studies described below illustrate how the work at NAYA provides continuity and support to $\mathrm{AI} / \mathrm{AN}$ emerging adults as they enter and move through the transition process; all of the studies were reviewed and approved by the university IRB committee. These studies were guided by the following research questions: (1) What are the transition needs and experiences of urban AI/ AN youth, and (2) What are the culturally based services provided to address these needs?

\section{Study 1: Identifying Culturally Based Inter- ventions}

Purpose To develop an understanding of the culturally responsive interventions provided to $\mathrm{Na}$ tive American youth at NAYA.

Method. Initially, members of the research team conducted a textual analysis of organizational documents to understand the ways that NAYA services were described in both internal and public documents. ${ }^{36}$ Systematic review yielded themes and categories for further inquiry. Based on this information, the researchers conducted a nominal group process with NAYA management and program directors. ${ }^{37}$ Using a process of brainstorming, categorizing, and consolidating, the researchers generated initial lists of strategic interventions organization wide. These were tested for accuracy with the key informants, and with NAYA's elders and board of directors. NAYA's board refined and confirmed the lists. Finally, in focus groups of youth, families, staff, elders, and service providers, participants were asked what NAYA does to help; these data were examined for alignment with the list of strategic interventions. Focus group methodology was selected for its demonstrated advantages in research with diverse population, including the potential to raise participants' awareness of the topic being studied, reduce the power differential between researchers and participants, and empower participants. ${ }^{38,39}$ Together, members of the research team sorted the strategic interventions around the RWV using a content analysis strategy, ${ }^{36}$ and the results were presented and discussed with NAYA staff to affirm that the research team's work fairly reflected the program's reality.

Findings. This effort yielded a list of 23 clusters of activities called "strategic interventions" that are used by staff at NAYA to address the needs of AI/ AN youth, including those in the transition years. These interventions are listed in Fig. 1. When members of the research team compared the 15 objectives of PYD presented by Catalano ${ }^{34}$ to NAYA's strategic interventions, all 15 of the PYD objectives were reflected, but the NAYA strategic interventions included some areas not directly addressed by PYD. These were: Mitigating the negative effects of 
racism; cultural preservation; holistic response; safe milieu, schools, and neighborhoods; case management; family support and interaction; and social justice and equity protection.

Summary. This study provided NAYA with a more systematic way of framing its culturally appropriate approaches to supporting AI/AN youth. The study team identified 23 strategic interventions utilized by NAYA, and placed those interventions within the RWV to create a framework that grounds practice. This framework was found to be similar to PYD, but included distinct culturally appropriate additions to its objectives.

\section{Study 2: Focus Groups and Individual Inter- views with $\mathrm{AI} / \mathrm{AN}$ Young Adults}

Purpose. To explore transition needs of urban Native youth, the challenges they faced, and their perceptions of what helped to support their positive transitions to adulthood.

Methods. Members of the research team conducted 5 focus groups and 9 individual interviews with 15 young women and 3 young men with foster care experience and 6 young women and 9 young men who had never been in foster care but who reported receiving mental health services. Focus group methods were selected for their potential to yield rich data on the shared experiences of a

Figure 1. Strategic interventions organized by RWV

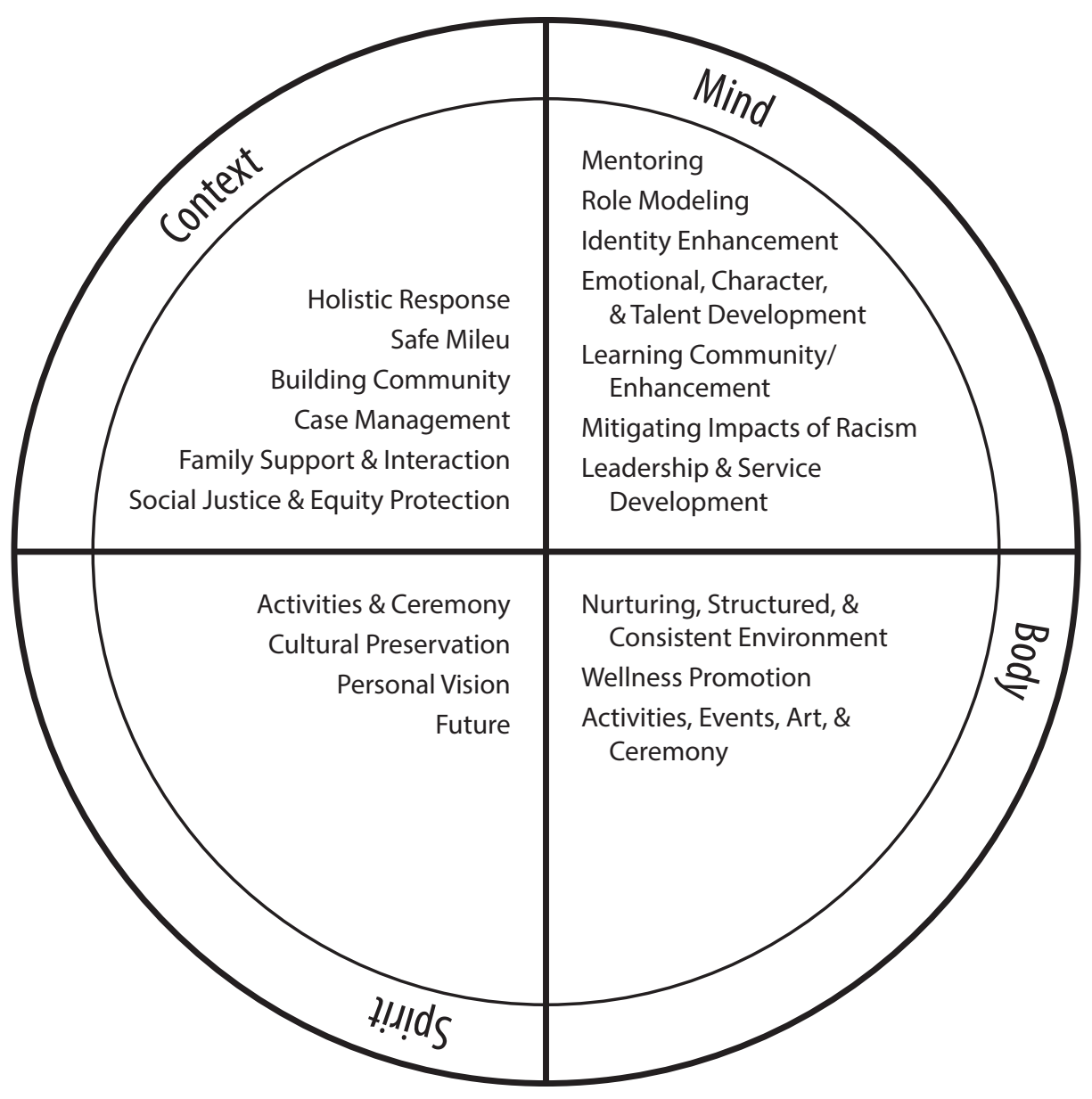


particular group of individuals, in this case youth in transition to adulthood, and to provide the research team and NAYA with a range of perspectives about the transition experience in the words of the youths themselves. ${ }^{39}$ Participants who identified as having had formal contact with mental health services had the choice of participating in individual interviews or focus groups. All participants were receiving services at NAYA, self-identified as Native American, and were 17-23 years old. In the focus groups and interviews, these young adults were asked to describe the challenges they encountered in the transition to adulthood and the services and supports that were most helpful. Based on guidance from agency staff, the research team did not audiorecord interviews, but took detailed notes, including direct quotes. Data were member checked with youth at the end of each interview to increase credibility of findings, as recommended by Creswell, ${ }^{40}$ and changes were made based on youth feedback. The member checking process most often resulted in youth providing clarifying statements to research staff about the meaning behind their comments which resulted in richer descriptions about these youth's transition experiences. Participants signed informed consent and were compensated with US $\$ 25$ gift cards.

Data analysis. Research team members transcribed all notes, and independently engaged in content analysis to identify themes that arose from focus groups and individual interviews. ${ }^{36}$ Research team members then met to compare and revise codes in order to increase inter-rater reliability. ${ }^{40}$

Themes were then sorted into the quadrants of the RWV model, and findings were presented to the team and NAYA staff in order to review codes, to ground the findings in the cultural context, and to clarify cultural understanding. Categories of findings situated within the RWV included the transition challenges described by young adults and the characteristics of helpful people and programs, both illustrated by brief representative quotes.

Findings. In the Context quadrant, participants identified experiences of traumatic events such as homelessness and fights with roommates, as well as challenges in getting support from the community, including how to break away from gang involvement. For example, "As long as nobody gets hurt, it's okay. If I could do it [make money] legally, that would be great." Young adults were challenged by not having support: "If I had better guidance from an adult, I probably would have come out of the other side [of transition] a little better." Participants also expressed their needs for positive relationships with peers and support professionals, and for their family to believe in their ability to succeed. For young parents, there were challenges in parenting, and for foster youth, in renegotiating relationships with parents (Context).

Participants reported challenges related to their desire to feel responsible, the need to build selfesteem and confidence, and to gain knowledge and skills. Other reported challenges included fear of becoming an adult, achieving an appropriate gender role, and working to achieve a balance between help-seeking and independence: "It's a continuing challenge between how much I put on myself and when to ask for help" (Mind).

Basic needs such as getting a job and apartment were reported as important, as well as self-care: "I had to find the inner strength to get sober and take care of my mental health" (Body). Finally, finding balance between freedom and responsibilities was reported as important, as well as cultural pride and spiritual beliefs: "Having spiritual beliefs for me has helped out" (Spirit).

In response to questions about what helped their transitions to adulthood, participants focused on relationships with families, foster parents, service providers, friends, mentors, and several NAYA programs. A recurring theme was the benefits of honesty and persistence in relationships: "My [foster] dad helped me the most out of everybody. He kept me out of trouble. Stayed with me through my teenage years when I was using alcohol and doing stupid stuff" (Context). Many participants expressed appreciation for NAYA staff's emotional support to recover from traumatic experiences and opportunities for skill building: "[NAYA advocate] just being on my back, making me do stuff, like handling my business, like school, making sure I'm turning in my homework and have good attendance. Making sure 
I get to my meetings" (Mind). Specific types of practical support included NAYA's providing homeless services, a Rent Well program, transportation to work and job interviews, food, clothes, toys, books, and the support of an independent living worker: "Letting me know about resources and knowing they were there even if I didn't use them. Helping make phone calls to college financial aid offices and walking me through steps to make it less scary. Being accountable to someone. Helping figure out scholarships" (Body). These participants appreciated the importance of their relatives' and NAYA staff teaching them about their culture and spirituality: "My culture is sometimes what keeps me going" (Spirit).

Summary. Participants who were transitioning out of foster care as well as those recruited because of their history of using mental health services said they were equally comfortable discussing their experiences in either research setting (focus groups or interviews). All participants described major challenges in all life domains of the RWV. While this brief summary provides only a few examples of their many reported challenges and helpful supports, there were consistent themes related to the safe and culturally supportive milieu of NAYA. These young people appreciated the warm relationships, consistency, exposure to opportunities for skill development, access to needed resources, and strength-orientation, along with high expectations to learn about and actively participate in the Native community and its cultural activities. As young people talked about their experiences at NAYA, the holistic, interconnected, and complex aspects of culturally based staff interventions and milieu activities in all four quadrants of the RWV were apparent.

\section{Study 3: Observation of the Youth Advocacy Process}

Purpose. The purpose of this study was to explore the elements of culturally based services supporting urban Native young people to make positive transitions to adulthood by examining the youth advocacy/case management process at NAYA.

Methods. The research team selected an eth- nographic approach using observation and ethnographic interviewing to examine and describe youth advocate interactions with young adults in youth advocacy/case management sessions in the natural settings in which this work is typically conducted. An ethnographic approach was selected because of its utility in enabling researchers to explore and describe social and cultural phenomena. ${ }^{36}$ Members of the research team developed an observational guide that listed the 23 strategic interventions and their sub-elements identified in Study 1 to observe and document case management/youth advocacy sessions in 5-min segments. They also developed semistructured interview protocols to use in individual interviews with young adults and youth.

Participants. Case management sessions involving two youth advocates and four young adults were observed in order to learn about the interventions and relationships that contributed to a positive transition to adulthood and the challenges AI/ AN young people face as they are involved in transitioning to adulthood. Both youth advocates were female and all 4 youth participants were female (due to difficulty in scheduling young men), aged 17-24. All participants were members of indigenous groups.

Data collection. First, researchers met with youth advocates who had been nominated by program leaders because of their expertise in providing culturally responsive services to young adults to become acquainted and to explain the purpose of the study. These youth advocates identified young adults with whom they had worked for at least 6 months and invited them to participate. Informed consent was obtained from both youth advocates and young adults prior to data collection. Members of the research team separately observed 14 youth advocacy sessions for up to an hour, utilizing the observation checklist while making notes about specific strategic interventions. The sessions occurred either at the NAYA community center or in a community location. In separate, brief, semistructured interviews after each session, researchers asked youth advocates open-ended questions about the specific strategic interventions they had used. They also asked both young people and youth 
advocates what elements of the session had been most helpful in meeting young people's needs. Interviews were digitally recorded and transcribed; for observations in noisy community settings, researchers noted verbatim responses of participants.

Data Analysis. Observational notes, transcripts, and interview notes were examined to identify patterns in the use of strategic interventions and examples of comments about the helpfulness of specific interventions in contributing to positive outcomes. Using a content analysis strategy, ${ }^{36,41}$ researchers noted themes in interview transcripts across sessions for each young person and youth advocate, then analyzed data across all interviews and prepared a summary of the interventions observed across the RWV quadrants and illustrated by representative interview quotes.

Findings. Youth advocates were observed to apply holistic and comprehensive approaches in their work, using clusters of strategic interventions. The following summary provides examples of frequently observed interventions that occurred together, illustrated by related interview quotes from emerging adults or youth advocates. Learning community/enhancement, mentoring, and role modeling (Mind): Youth advocates and young people focused on learning enhancement while advocates provided mentoring and role modeling for youth to develop and implement educational plans and prepare for other important transitions. For example, a young woman described her youth advocate's mentoring for school success: "When I do need help I know I can talk to her and she'll help me. Support with whatever I need, schoolwork, or whatever I need. Definitely encourages me. She knows what's going on." Future orientation (Spirit) and maintain high expectations and standards (Context): Youth advocates set high expectations and conveyed their beliefs that these young people had the skills, strengths, and abilities to attain their goals for adulthood, as noted by a youth advocate: "Her skill set is so high.... It's not really a lot about building those skill sets with her, but just about getting her to think about making sure her plan and goals are getting there."

Another cluster of strategic interventions observed included Nurturing, structured, and consistent environment (Body) and safe milieu (Context). Responding to the high levels of trauma experienced by youth served at NAYA, youth advocates reported striving to create a consistent and a safe environment and youth expressed that they felt safe to discuss traumatic experiences and problems. For example, a young woman reported her sense of safety: "She's been in at the beginning of my journey - this chaotic mess - and taking me out of a DV home to a homeless and pregnant mom. I really trust her.... She's a friend because she cares about me. She knows about me as a person." Cultural preservation (Spirit), identity enhancement, and emotional development (Mind): During the observations, these young people were dealing with many challenges, such as homelessness, lack of resources, and family conflict, so the youth advocacy sessions were less focused on specific activities to enhance identity and preserve culture. Even so, young adult participants described their sense of cultural connection and belonging at NAYA and appreciation for the opportunity to work with a youth advocate from the same cultural background as they struggled with their issues. For example, a young woman commented: "I think that knowing that she's [youth advocate] in a similar cultural background, it make me feel safer to address my needs. I know that she can understand not just like my situation, but like the cultural background that I have that affects my decisions when I do things."

Summary. The findings of this study illustrated the holistic nature of interventions at NAYA. These young people were dealing with complex challenges that had continued or recurred over time and the youth advocates had been pursuing an array of interventions with them for at least several months. Youth advocates were observed to utilize a range of intervention strategies across the RWV usually in combination, rather than discrete strategies, making it difficult for observers to dissect activities. Attention to safe, consistent, and nurturing relationships was observed in all sessions, with focus on future orientation, strengths, and high expectations, especially with regard to educational success and positive transitional outcomes. Culturally compatible youth advocates demonstrated understanding of 
the young people's cultural background and needs, as well as caring and commitment to resolving youth-defined challenges. Young people expressed appreciation for the ongoing support of their youth advocate and for gaining access to resources, opportunities, and cultural connections.

A limitation of this study was that the focus on youth advocacy sessions did not formally examine the cultural milieu of NAYA, which included support from elders and other community members and opportunities for culturally based activities and leadership opportunities. Additionally, the presence of an outside observer has the potential to shift the dynamic between youth advocate and the young person during their case management session. Finally, the time-limited study was unable to capture linkages between the utilization of strategic interventions and positive transition outcomes.

\section{Discussion}

These findings of the three studies presented here reflect multiple voices that describe how a culturally based community agency works to support Native American youth in the transition to adulthood. Study 1, which drew from the perspectives of youth, families, staff, elders, and service providers, resulted in a refined list of the interventions used within an overall holistic approach to supporting AI/AN youth. The study identified 23 strategic interventions that are used by staff across services to support youth in transition to gain harmony and balance across the four quadrants of the RWV. The findings from this study provided a foundation for the next two studies. In Study 2, researchers heard directly from young people in transition who have experienced much hardship (e.g., mental health conditions, homelessness, foster care, gang membership, and/or substance abuse). These emerging adults identified challenges and supports across all four RWV quadrants. Finally, Study 3 incorporated the voices of young people who had mental health challenges and their culturally matched youth advocates. This study revealed that advocates use culturally grounded, comprehensive approaches to support positive transitions. These approaches were often seen in recurring clusters of strategic interventions that bridge quadrants of the RWV. Taken together, these findings suggest that to address the mental health and well-being of AI/AN youth in culturally appropriate ways, the needs of the young person should be reviewed across all domains of the RWV—context, mind, body, and spirit. These results demonstrate the intentional application of culturally grounded interventions to overcome the traumatic histories and current oppressive conditions affecting low-income urban Native American youth with mental health challenges, and to support their wellbeing and positive transition to adulthood.

Limitations of these studies are related to the use of qualitative research methods, which involved small samples of participants in one urban agency setting, thereby limiting the potential for generalization. Additionally, the descriptive reports of intervention strategies, challenges and supports, and observations of interactions over the short-term could not be linked with outcomes. While young people talked positively about culturally based support they had received in the focus groups and interviews, the researchers were unable to determine the specific aspects of culturally grounded interventions that effectively addressed their mental health needs or contributed to positive transition outcomes. Finally, the research was conducted by a team that included Native and non-Native researchers and service providers. Although the potential for cultural misunderstandings was addressed through extensive and frequent de-briefing sessions throughout the research process, it is likely that content and meaning were lost in these cross-cultural transactions. Despite these limitations, these studies add to the knowledge base about the needs of $\mathrm{AI} / \mathrm{AN}$ emerging adults, and contribute a beginning understanding of culturally grounded interventions designed to promote their successful transitions to adulthood. Understanding of the effectiveness of this approach will also be extended through research currently being conducted at NAYA to measure the community's culturally defined transition outcomes using the culturally grounded assessment tool developed by the research team.

To better understand the impact and outcomes of the whole array of strategic interventions on 
Native youth development over time, future research will need to take into account the cultural context of culturally based service settings, and to understand the impact of services and supports from the perspective of the AI/AN young person. Perhaps such research strategies would include following youth across all of the settings and activities available in the milieu. This "following" could be done literally, or through strategies such as electronic checklists and note-taking by the young people themselves.

\section{Implications for Behavioral Health}

The studies reported here were conducted within one specific culturally based community agency serving AI/AN youth and their families, which may be unique even among other such organizations. It is difficult to imagine that dominant culture community-based agencies could provide the same culturally intensive, welcoming, positive, safe, and consistent milieu as that observed at NAYA. Ideally, then, all AI/AN emerging adults with mental health concerns would have access to the same degree of cultural understanding; high expectations; role modeling; and emotional, tangible, and social support as that provided at NAYA. Such resources, however, are not available in all communities, and it is unlikely that there are enough culturally specific resources for all AI/AN young people in need. This raises the question of what mainstream mental health and other youth-serving organizations, practitioners, and policymakers can do to promote the positive mental health and successful transition of $\mathrm{AI} / \mathrm{AN}$ emerging adults.

The first recommendation is for mainstream organizations, practitioners, and policymakers to acknowledge the unique transition challenges of urban AI/AN youth and the potential power of culturally based services to support them through a complex transition process. The implications of this recommendation are several. First, individual AI/AN youth and families should be connected to culturally specific services whenever possible, and referrals to these services should be reinforced with vigorous efforts to ensure that the intended connections occur. At the organizational level, employing cultural consultants with strong community connections and knowledge of existing resources for AI/AN youth can help to facilitate this process; they can also provide ongoing consultation to practitioners, administrators, and board members regarding the $\mathrm{AI} / \mathrm{AN}$ youth and families that they serve. And, of course, recruitment and support of $\mathrm{AI} / \mathrm{AN}$ staff at all organizational levels is essential.

Mainstream organizations and policymakers are also encouraged to seek partnerships with culturally based organizations to undertake activities that will enhance services for AI/AN youth and families to the mutual benefit of all partners. Some suggestions for actions are provided by the Center for Reducing Health Disparities at UC Davis, ${ }^{42}$ including a guide to community engagement. After conversations with Native American leaders, service providers, and youth about mental health and the Native American community, Center personnel worked with state policymakers to promote policy change directed toward addressing the needs of underserved communities; facilitated the involvement of AI/AN communities in county and state level decision-making processes; and, helped Native communities identify opportunities to obtain funding for needed programs.

It may be difficult for many mainstream mental health programs to appropriately serve AI/AN youth with serious mental health conditions. Bigfoot $^{43}$ refers to Manson's observation that tensions related to the mental health system and AI/AN people include language (e.g., use of the term "mental illness" when AI/AN people embrace wellness), and concepts such as etiology and treatment contrasted with an AI/AN emphasis on health promotion. This does not mean that the specialized knowledge and training of mainstream mental health providers is of no value to $\mathrm{AI} / \mathrm{AN}$ people, but it does suggest that innovative practice approaches need to be developed, based on an understanding of the cultural context of the emerging adults to be served. These shifts in practice can include partnering with traditional Native providers to conduct assessments and develop service plans and developing teams of Native and non-Native consultants to increase cross-cultural (mainstream mental health and $\mathrm{Na}-$ tive) understanding at interpersonal, practice, and organizational levels. 
Some of the differing views about mental health mentioned above are also reflected in the mental health recovery research and other literature. ${ }^{4-46}$ This body of literature, along with providers and persons in recovery, may also be a source for generating expanded practice and policy approaches to working with AI/AN people. Many of the recommendations for mental health practitioners and organizations seeking to promote recovery-oriented organizations and practice may be useful, e.g., shifting from expert to consultative models, acknowledging the important of customer choice, attending to organizational and system barriers, and others.

Of existing mainstream programs, Positive Youth Development models reflect principles that are highly compatible with the values and principles found in practice at NAYA. Several authors address the compatibility of PYD principles with AI/AN values and teachings, ${ }^{35,47}$ and there may be promise in the exchange of ideas and information here, as well. PYD research and practice have much to offer culturally based programs, and PYD theorists could stretch their concepts and practice to become even more culturally relevant. A future challenge, then, will be to blend useful elements from the holistic and comprehensive approach exemplified by NAYA, valuable knowledge from mainstream mental health research and practice, and the contributions of PYD to address the challenges faced by AI/ AN youth as they transition into adulthood across two cultures.

\section{Conflict of Interest}

None of the authors reports a conflict of interest.

\section{Acknowledgments}

This work was supported by funding from the National Institute of Disability and Rehabilitation Research, United States Department of Education, and the Center for Mental Health Services, Substance Abuse and Mental Health Services Administration, United States Department of Health and Human Services (NIDRR grant H133B090019), and the Office of Juvenile Justice and Delinquency Prevention, United States Department of Justice,
Office of Justice Programs (OJJDP grant 2011-TYFX-0103).

The authors also want to thank all members of the NAYA community that participated in this research effort. Thanks to the NAYA elders and board for their interest and wisdom, and to the young people and staff from NAYA for generously sharing their stories, their lives, and their time. It is our hope that their gifts will result in a better understanding of how to support Native youth who are approaching or in the midst of the transition process.

\section{References}

1. Gone JP. Encountering professional psychology: Re-envisioning mental health services for Native North America. In: LJ Kirmayer, G Valaskakis (Eds). Healing Traditions: The Mental Health of Aboriginal Peoples. Vancouver BC: University of British Columbia, 2009, pp. 419-439.

2. Stiffman AR, Brown E, Freedenthal S, et al. American Indian youth: Personal, familial, and environmental strengths. Journal of Child and Family Studies 2007; 16: 331-346.

3. Goodkind J.R., Ross-Toledo K, John S, et al. Promoting healing and restoring trust: Policy recommendations for improving behavioral health care for American Indian/Alaska Native adolescents. American Journal of Community Psychology 2010; 46: 386-394.

4. Urban Indian Health Institute, Seattle Indian Health Board. Urban American Indian and Alaska Native Youth: An Analysis of Select National Data Sources. Seattle, WA: Urban Indian Health Institute, 2009.

5. Zhang D., Katsiyannis A., Ju S., et al. Minority representation in special education: 5-year trends. Journal of Child and Family Studies 2014; 23: 118-127.

6. Duran E, Duran B. Native American Postcolonial Psychology. Albany, NY: State University of New York Press, 1995.

7. Yellow Horse Brave Heart M, Chase J, Elkins J, et al. Historical trauma among indigenous peoples of the Americas: Concepts, research, and clinical considerations. Journal of Psychoactive Drugs 2011; 43(4): 282-290.

8. Walls ML, Whitbeck LB. The intergenerational 
effects of relocation policies on Indigenous families. Journal of Family Issues 2012; 33(9): 1272-1293.

9. Ehlers CL, Gizer IR, Gilder D, et al. Lifetime history of traumatic events in an American Indian community sample: Heritability and relation to substance dependence, affective disorder, conduct disorder and PTSD. Journal of Psychiatric Research 2013; 47(2): 155-61.9.

10. DeVoe JF, Darling-Churchill KE, Snyder TD. Status and Trends in the Education of American Indians and Alaska Natives: 2008. Washington, DC, 2008.

11. Long C, Downs AC, Gillette B, et al. Assessing cultural life skills of American Indian youth. Child \& Youth Care Forum 2006; 35(4): 289-304.

12. Ramasamy R. Post-high school employment: A follow-up of Apache Native American youth. Journal of Learning Disabilities 1996; 29(2): 174-179.

13. Ramasamy R, Duffy M, Camp JL. Transition from school to adult life: Critical issues for Native American youth with and without learning disabilities. Career Development Transition and Exceptional Individuals 2000; 23(2): 157-171.

14. Shafer MS, Ramasamy R. Transition and Native American Youth: A follow-up study of school leavers on the Fort Apache Indian Reservation. Journal of Rehabilitation 1995; 61(1): 60-65.

15. Leake DW, Black RS, Roberts K. Assumptions in transition planning: Are they culturally sensitive? Impact 2003; 4(1): 28-29.

16. Cross TL. The Relational Worldview and child well-being. Child Welfare 360. Center for Advanced Studies in Child Welfare, St. Paul: University of Minnesota (2014, in press).

17. Cross TL. Understanding family resiliency from a relational world view. In: HI McCubbin, EA Thompson, AI Thompson, et al., (Eds.). Resiliency in Native American and Immigrant Families. Thousand Oaks, CA: Sage, 2008, pp. 143-157.

18. Hodge DR, Limb GE, Cross TL. Moving from colonization toward balance and harmony: A Native American perspective on wellness. Social Work 2009; 54(3): 211-19.

19. Cross T, Friesen BJ, Jivanjee, et al. Defining youth success using culturally appropriate communitybased participatory research methods. Best Practices in Mental Health 2011; 7(1): 94-115.
20. Gowen LK, Bandurraga A, Jivanjee P, et al. Development, testing, and use of a valid and reliable assessment tool for Urban American Indian/Alaska Native Youth programming using culturally appropriate methodologies. Journal of Ethnic Cultural Diversity in Social Work 2012; 21(2): 77-94.

21. About NAYA. Available online at http://nayapdx. org/about/. Accessed November 15, 2013.

22. Chandler M, Lalonde C. Cultural continuity as a moderator of suicide risk among Canada's First Nations. In: L Kirmayer, G Valaskakis, (Eds). Healing Traditions: The Mental Health of Aboriginal Peoples in Canada. Vancouver, BC: University of British Columbia Press: 2009, pp. 221-248.

23. Feliciano C. The benefits of biculturalism: Exposure to immigrant culture and dropping out of school among Asian and Latino youths. Social Science Quarterly 2001; 82(4): 865-879.

24. Whitbeck LB, Hoyt DR, Stubben JD, et al. Traditional culture and academic success among American Indian children in the upper Midwest. Journal of American Indian Education 2001; 40(2): 48-60.

25. Jones M, Galliher R. Ethnic identity and psychosocial functioning in Navajo adolescents. Journal of Research on Adolescence 2007; 17(4): 683-696.

26. Kulis S, Napoli M, Marsiglia FF. Ethnic pride, biculturalism, and drug use norms of urban American Indian adolescents. Social Work Research 2002; 26(2): 101-112.

27. Herman-Stahl M, Spencer D, Duncan J. The implications of cultural orientation for substance abuse use among American Indians. American Indian Alaska Native Mental Health Research 2003; 11(1): 46-66.

28. Schinke SP, Tepavac L, Cole KC. Preventing substance use among Native American youth. Addiction Behavior 2000; 25(3): 387-397.

29. LaFromboise TD, Hoyt DR, Oliver L, et al. Family, community, and school influences on resilience among American Indian adolescents in the upper Midwest. Journal of Community Psychology 2006; 34(2): 193-209.

30. LaFromboise TD, Coleman HL, Gerton J. Psychological impact of biculturalism: Evidence and theory. Psychology Bulletin 1993; 114(3): 395-412.

31. Wexler L. The importance of identity, history, and culture in the wellbeing of indigenous youth. The 
Journal of the History of Childhood and Youth 2009; 2(2): 267-276.

32. Blackstock $\mathrm{C}$. The emergence of the breath of life theory. Journal of Social Work Values and Ethics 2011; 6(3): 13-2.

33. Curry-Stevens A, Cross-Hemmer A, Coalition of Communities of Color. The Native American Community in Multnomah County: An Unsettling Profile. Portland, OR: Portland State University; 2011.

34. Catalano RF, Berglund ML, Ryan JAM, et al. Positive youth development in the United States: Research findings on evaluations of positive youth development programs. Annals of the American Academy Political and Social Science 2004; 591(1): 98-124.

35. Cheshire TC, Kawamoto WT. Positive youth development in urban American Indian adolescents. In: FA Villarruel, DF Perkins, LM Borden, et al., (Eds.). Community Youth Development. Thousand Oaks, CA: Sage, 2003, pp. 79-89.

36. Hesse-Biber S, Leavey P. The Practice of Qualitative Research. Thousand Oaks, CA: Sage Publications, 2006.

37. Delbecq AL, Van de Ven AH. A group process model for problem identification and program planning. The Journal of Applied Behavioral Science 1971; 7(4): 466-492.

38. Linhorst DM. A review of the use and potential of focus groups in social work research. Qualitative Social Work 2002; 1(2): 208-228.

39. Ivanoff SD, Hultberg J. Understanding the multiple realities of everyday life: Basic assumptions of focus-group methodologies. Scandinavian Journal of Occupational Therapy 2006; 13: 125-132.
40. Creswell JW. Qualitative Inquiry \& Research Design: Choosing Among Five Approaches, 2nd Edition. Thousand Oaks, CA: Sage Publications, 2007.

41. Grbich C. Qualitative Data Analysis: An Introduction. Thousand Oaks, CA: Sage Publications, 2010.

42. UC Davis Center for Reducing Health Disparities. Building Partnerships: Conversations with Native Americans about Mental Health Needs and Community Strengths. Sacramento, CA: Center for Reducing Health Disparities, 2009.

43. BigFoot DS. Cultural adaptations of evidence-based practices for American Indian and Alaska Native populations. In: C Newman, CJ Liberton, $\mathrm{K}$. Kutash, et al. (Eds.). A system of care for children's mental health. Tampa, FL: University of South Florida, Louis de la Parte Florida Mental Health Institute, 2008, pp. 69-79.

44. Gehart DR. The mental health recovery movement and family therapy, Part I: Consumer-led reform of services to persons diagnosed with severe mental illness. Journal of Marital \& Family Therapy 2012; 38(3): 429-442.

45. Russinova Z, Rogers ES, Ellison ML, et al. Recovery-promoting professional competencies: Perspectives of mental health consumers, consumerproviders, and providers. Psychiatric Rehabilitation Journal 2011; 34(3): 177-185.

46. Sowers W. Transforming systems of care: The American Association of Community Psychiatrists guidelines for recovery oriented services. Community Mental Health Journal 2005; 42(6): 757-774.

47. Garrett, M, Parrish, M, Williams, C, et al. Invited Commentary: Fostering resilience among Native American youth through therapeutic intervention. Journal of Youth and Adolescence 2014; 43:470-490.

This manuscript was published online November 12, 2014 in the Journal of Behavioral Health Services \& Research. The final publication is available at Springer via http://link.springer.com/article/10.1007/s11414-014-9447-2

This activity is supported by a grant funded by both the National Institute of Disability, Independent Living, and Rehabilitation Research, and the Center for Mental Health Services Substance Abuse and Mental Health Services Administration, United States Department of Health and Human Services (NIDILRR grant 90RT5030). NIDILRR is a Center within the Administration for Community Living (ACL). The content does not necessarily represent the policy of NIDILRR, ACL, HHS, and you should not assume endorsement by the Federal Government.

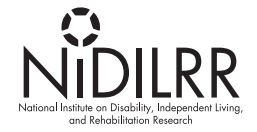

SAMHSA 